\title{
Inversion of Many-beam Bragg Intensities for Phasing by Iterated Projections : Removal of Multiple Scattering Artifacts.
}

\author{
John Spence ${ }^{1}$ and Jeffrey Donatelli ${ }^{2}$ \\ ${ }^{1}$ Arizona State University, Tempe, Arizona, United States, ${ }^{2}$ Lawrence Berkeley Laboratory, Berkeley, \\ California, United States
}

An iterated projection algorithm (N-Phaser) is demonstrated which solves the inversion problem of Nbeam multiple scattering from Bragg intensities [1]. Since these intensities result from coherent interference between beams, this solves the phase problem. We demonstrate the recovery of 840 complex structure factors from 441 simultaneously interacting Bragg beams for an acentric crystal structure in high energy transmission electron diffraction (TED), where the algorithm recovers accurate complex structure factors from a wide range of thicknesses, orientations and relativistic beam energies. The sample thickness need not be known. Backscattering and polarization (present for X-rays) are ignored in this scalar theory. The method may be used to eliminate multiple scattering artifacts from diffraction data (reducing $R$ factors), and to increase the thicknesses of samples used in structural biology and materials science.

Multiple elastic scattering in X-ray, electron and neutron diffraction prevents extraction of useful structure factors, but may be useful for standing wave generation (to modulate fluorescence), to provide sensitivity to chirality, and for space-group determination (by removing Friedel symmetries). The thickness limit which multiple scattering imposes on samples severely hampers, for example, the effort toward whole-cell imaging in cryo-electron microscopy, the size of protein microcrystals in the new TEM method of MicroED, sample thickness in X-ray Diffractive Imaging, and the thickness of the crystals and their defects studied by atomic-resolution electron microscopy.

Inversion from multiple to single scattering solves the phase problem. Few-beam cases have been used successfully for phasing hard X-ray inorganic crystal diffraction, and the phase problem is an urgent issue in the new field of MicroED, where protein microcrystal structures are solved by TED with Cryo-EM instrumentation involving thousands of simultaneously excited beams. Here Direct Methods for phasing are limited by the number of atoms in the molecule, while Molecular Replacement depends on existing models. With the development of nanometer-focusing hard X-ray lenses, a solution to this problem would also provide an X-ray microdiffraction technique capable of producing phased lensless images of small regions of much thicker samples in materials science and biology.

The relationship between formulations of the multiple-scattering problem, including the Multislice, Darwin equations, Bloch-wave methods, the Scattering Matrix, Path Integral approaches, and their derivation from the Schroedinger equation is given elsewhere [2, 3]. Partially successful inversion has been based on closed-form solutions [4,5], on large- angle convergent-beam patterns [6], dynamical Ptychography [7], optical Multislice Ptychography [8], iterated projection algorithms [9], data collected at two adjacent beam energies $[10,11]$, and inversion from a scattering matrix $S$ [12]. Inversion from the complex multiply-scattered amplitudes which form atomic-resolution STEM images [13], using multiple detector pixels to provide simultaneous tilted images, is an important recent advance.

The scattering matrix solution to a relativistically corrected Schroedinger equation, describing highenergy transmission electron diffraction (HEED) through a slab of crystal of thickness $t$, is defined as $\mathbf{S}=$ $\exp (2 \pi \mathrm{iAt})$. Here $\mathbf{S}$ contains complex dynamical amplitudes (one diffraction pattern per column) for many 
orientations around a zone axis, while A contains wanted complex structure factors in off-diagonal positions and known excitation errors on the diagonal [7]. Absorption is included on the diagonal of A. Our new algorithm extends an earlier iterative projection approach [6] which projects between $\mathbf{S}$ and A with the addition of suitable constraints, by adding a potential-flipping step (used previously for phasing kinematic CBED patterns [14] ) and by addressing an instability which previously occurred for large thickness. Constraints include the known diagonal of $\mathbf{A}$ and its symmetries $[15,16]$. At small thickness, given atomic-resolution data, the flipping step alone phases the data. We now avoid diagonalizing $\mathbf{A}$ at large thickness to obtain $\mathbf{S}$, due to sensitivity to small changes in $\mathrm{t}$, using the known magnitude constraint on $\mathbf{S}$ to obtain its eigenvalue [1].

In the computational trials shown for GaAs [110] TED [1] with $\mathrm{N}=441$, apart from an origin shift, the algorithm correctly recovers the same complex structure factors at thicknesses of $10 \mathrm{~nm}, 100 \mathrm{~nm}$ and $1000 \mathrm{~nm}$, at beam energies of $100 \mathrm{kV}, 300 \mathrm{kV}$ and $1 \mathrm{MeV}$ despite widely different dynamical Bragg intensities. The Pearson correlation coefficient between the recovered and true potential is around 0.95, but decreases at energies above $1 \mathrm{MeV}$ where the algorithm fails (except in the small thickness limit) as all excitation errors tend to zero. The method may be extended to include 3D reflections in the HOLZ [3] and possibly also to non-periodic samples for Cryo-em single particle imaging, using Shannon, rather than Bragg angular sampling. Unlike methods which used interferometry to measure dynamical phases, such as HREM images [13] or Ptychography, the recovered structure factor phases are not sensitive to instrumental phases such as focus setting. By solving the phase problem, this approach would then provide a general method of lensless imaging.

\section{References}

[1] Donatelli J.J. and J.C.H. Spence. Phys. Rev. Letts. Submitted. (2020).

[2] Goodman, P., and Moodie A.(1974) Acta Cryst A30, 280

[3] Zuo , J. and Spence, J.C.H. Advanced TEM. Springer (2017).

[4] Moodie, A. F. (1972). Z. Naturforsch. 27a, 437-444.

[5] Koch, C. and Spence, J. (2003). J. Phys. A, 36, 803

[6] Wang, F., et al. Phys. Rev. Letts. (2016) 117, 1, 015501.

[7] Spence, J. C. H. (1998). Acta Cryst. A54, 7-18

[8] Maiden A.M. et al. (2012) JOSA A 29, no. 81606.

[9] Spence, J.C.H. et al (1999) Acta Cryst. A55, 112.

[10] Spence, J.C.H. (2009) Acta Cryst A65 2009[

11] Rez, P. (1999) Acta Cryst A55, 160 - 167.

[12] Sturkey, L. (1962). Proc. Phys. Soc. 80, 321.

[13] Brown et al Phys Rev Letts 121 , 266102 (2018)

[14] McKeown J. and Spence J. (2009) J. Appl Phys. 106, 074309.

[15] Allen L. J. et al 1998 Acta Cryst A54, p. 388

[16] Kogiso M.et al.(1977). J. Phys. Soc. Jap. 42, 223. 